\title{
Modelo para la mejora del desempeño organizacional a través de las prácticas de la gestión de la calidad, gestión del conocimiento y liderazgo transformacional en una universidad privada
}

\author{
${ }^{1}$ Carlos Mediver Coaquira Tuco ${ }^{\mathrm{a}}$ \\ ${ }^{1}$ Universidad Peruana Unión \\ ${ }^{1}$ ORCID ID: https://orcid.org/0000-0002-0845-0415
}

Recibido: 13 de enero de 2018

Aceptado: 11 de junio de 2018

\section{Resumen}

La presente investigación, tuvo la finalidad de validar las relaciones de tipo causal según la propuesta del modelo teórico explicando que la mejora del desempeño organizacional se desarrolla a través de las prácticas de gestión de calidad, gestión del conocimiento y liderazgo transformacional. Para ello, se aplicó un cuestionario a una muestra por conveniencia de 222 directivos y docentes de la universidad privada. El diseño de investigación fue considerado como correlacional múltiple con un enfoque transversal. Los datos se analizaron por medio de un sistema de ecuaciones estructurales. Los parámetros obtenidos para valorar la bondad de ajuste del modelo fueron los siguientes: $\mathrm{x}^{2}$ con un valor de $2463.580, \mathrm{x}^{2} / \mathrm{gl} \mathrm{de} 1.582$ menor o igual a 3.0, RMSEA de 0.051 menor o igual a .07, GFI de 0.736 mayor o igual a .70, y CFI de .903 mayor o igual a .90 , estos índices revelan una muy buena adaptación de la estructura y validación del modelo propuesto, así también todas las relaciones de tipo causal en el modelo ( $p$-valor < 0.05) son estadísticamente significativos. Con esto se afirma que el desarrollo del desempeño organizacional es afectado por las interacciones de las prácticas de gestión de calidad, gestión del conocimiento y liderazgo transformacional.
\end{abstract}

Palabras clave: Desempeño organizacional, gestión de calidad, gestión del conocimiento, liderazgo transformacional.

\section{Model for the improvement of organizational performance through the practices of quality management, knowledge management and transformational leadership in a private university}

\begin{abstract}
Abstrac
The purpose of this research study was to validate the causal relationships according to the proposed theoretical model, explaining that the improvement of organizational performance is developed through quality management practices, knowledge management and transformational leadership. To achieve this, a questionnaire was applied to a convenience sample of 222 administrators and teachers of a private university. The research design was considered as multiple correlational with a transversal focus. The data was analyzed by means of a system of structural equations. The parameters obtained to assess the goodness of fit of the
\end{abstract}

${ }^{a}$ Correspondencia al autor

E-mail: cmcoaquira@upeu.edu.pe 
model were the following: $\mathrm{x}^{2}$ with a value of $2463.580, \mathrm{x}^{2} / \mathrm{gl}$ of 1.582 less than or equal to 3.0, RMSEA of 0.051 less than or equal to .07 , GFI of 0.736 greater than or equal to .70 , and CFI of .903 greater than or equal to .90 , these indices reveal a very good adaptation of the structure and validation of the proposed model, as well as that all causal relations in the model ( $\mathrm{p}$-value $<0.05)$ are statistically significant. Thus it is affirmed that the development of the organizational performance is affected by the interactions of the practices of quality management, knowledge management and transformational leadership

Key words: Organizational performance, quality management, knowledge management, transformational leadership

\section{Introducción}

La alta complejidad y competitividad de las empresas, producto de cambios continuos, profundos y acelerados en un entorno globalizado, sumado a mayores exigencias de calidad de los productos y/o servicios por parte de los clientes, requiere de un máximo de eficacia y eficiencia en la dirección de las mismas (Arelis, 2016). En la actualidad las instituciones educativas de educación superior, se ven también como una empresa, que debe alcanzar niveles óptimos de desempeño, con la única y principal diferencia de que el producto, un profesional, que debe tener competencias, habilidades y destrezas para satisfacer las necesidades de un mundo globalizado y cambiante, presentándose así un desafío de complejidad mayor para la universidad.

Las universidades de alguna forma responden a las demandas y expectativas de una sociedad postmoderna excesivamente abrumadora. Sin embargo, sus métodos y formas resultan muy tradicionales y, en muchos casos, obsoletos para responder a los nuevos requerimientos, porque la velocidad de la respuesta, en muchas ocasiones, no satisface al mercado cada vez más exigente en sus demandas y exigencias, tal como lo expresa Alcántara y Arcos (2004, citado por Turpo J. 2014).

En la actualidad, existen en el Perú 142 universidades, 51 universidades públicas y 91 privadas lo que evidencia el incremento de universidades con respecto a lo existente en el año 2011, donde hubo 102 universidades, de los cuales 35 eran públicas y 67 privadas. De las 142 universidades en funcionamiento actual, 76 tienen autorización definitiva, 53 con autorización provisional y 13 con ley de creación (MINEDU, 2015). Tales cifras evidencian una explosión no controlada de instituciones y programas de formación de dudosa calidad que difícilmente preparan al profesional competitivo que el país requiere.

Uno de los grandes problemas que afronta el país es la carencia de datos cuantitativos o cualitativos que ilustren el desempeño organizacional de la universidad, ello se debe en buena cuenta a la ausencia de un sistema de aseguramiento de la calidad y, que a pesar de los importantes esfuerzos realizados por instituciones o de forma personal por algunos destacados investigadores, lo cierto es que dispone de una data insuficiente, que no cubre todos los campos del accionar universitario.

Siendo que la Universidad es considerada como un proceso de formación mediante el cual un estudiante ingresante, insumo de este proceso, se transforma en un egresado, con competencias que les permita una mayor y mejor inserción laboral, favoreciendo su acceso a empleos formales y de calidad, la universidad se ve obligada a enfrentar el desafío de insertarse en un mundo complejo, con nuevas exigencias de profesionalismo y competencias emergentes asociadas a la sociedad del conocimiento; a ello se suman las nuevas tareas pedagógicas, y los requerimientos de rendir cuentas y ser eficientes en la gestión institucional (Espinoza, 2015).

Por otro lado, a nivel teórico, los intentos de brindar luces sobre esta problemática han procedido fundamentalmente del área de gestión de las organizaciones, proponiéndose diferentes modelos teóricos evaluados y validados al sustentar que la mejora del desempeño organizacional es afectada a través de las prácticas de gestión de calidad (Hussain y Younis 2015; Lee, 2015; Kiprotich, 2014; Abu- Jarour, 2013; Munizu 2013). Además, otros estudios han revelado la relación directa que existe entre la gestión del conocimiento y el desempeño organizacional (Salami y Obgeta 2015; Rasula, Bosilj y Indihar, 2012; McKeen, Zack y Singh 2006). Así también, se demostrado que el liderazgo transformacional y la cultura organizacional afectan positivamente en el desempeño organizacional (Kamarulzaman y Mohamed, 2016; Hassan, Shaukat y Saqib, 2013; García, Jiménez y Gutiérrez 2012; García, Lloréns y Verdú, 2007), así como en el desempeño laboral de los empleados de una organización (Jyoti y Bhau, 2015). 


\section{Gestión de la Calidad}

La Gestión de Calidad son un conjunto de acciones que orientan a planificar, organizar y controlar la función imprescindible para establecer la calidad en una organización (Pola, 1999). Según Ruiz \& López (2004) la Gestión de Calidad tiene impacto estratégico en la empresa y representa una oportunidad para generar competitividad, poniendo énfasis en el mercado y en las necesidades del cliente.

Por otro lado, Caballano (2012) afirma que la gestión de calidad es una filosofía adoptada por organizaciones que confían en el cambio orientado hacia el cliente y que persiguen mejoras continuas en sus procesos diarios. Esto implica que su personal también puede tomar decisiones.

El concepto de calidad ha ido evolucionando desde sus orígenes, aumentando objetivos y cambiando sus orientaciones hacia la satisfacción plena de los clientes. Comenzó como una necesidad de controlar e inspeccionar hasta llegar a lo que hoy conocemos como por calidad total, también denominado como excelencia. La evolución histórica de la gestión de la calidad ha estado llena de estudios y puesta en marcha de sistemas de gestión, herramientas y técnicas, impulsados por gurús de la calidad.

Ahora, consideramos pertinente señalar que Saad y Siha (2000) has distinguido cinco etapas en la evolución de la calidad.

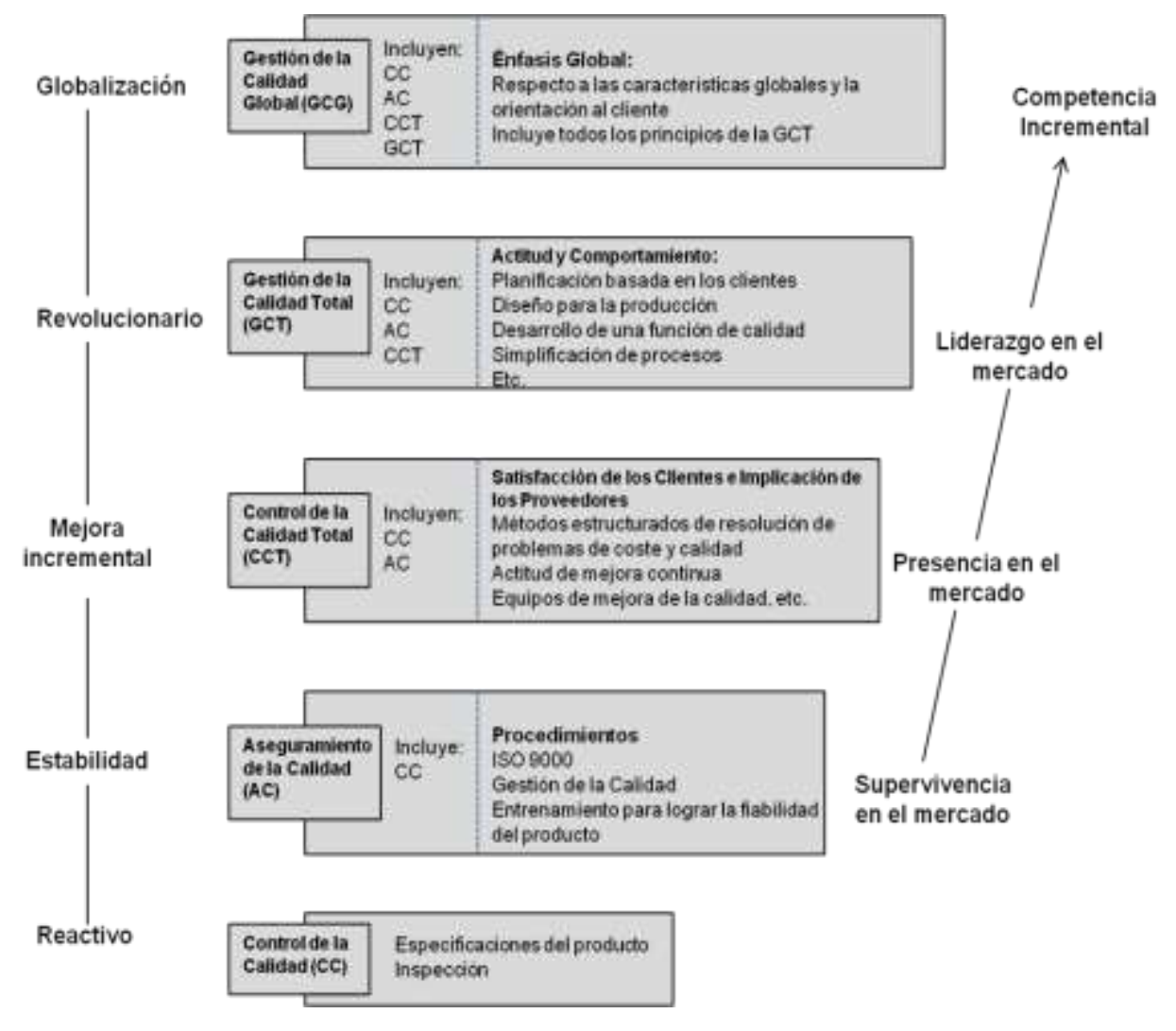

Figura 1. Etapas en la evolución de la gestión de la calidad según Saad y Siha (2000).

Saravanan y Rao (2006) sostienen que es necesario identificar y validar las dimensiones críticas de la gestión de la calidad en las empresas de servicios y, por tanto, no se puede hacer una simple traslación de lo ejecutado en la industria a organizaciones de servicios. Así pues, en este estudio se consideró plantear dimensiones que presentan un mayor consenso en la literatura y que han sido validadas en empresas del sector servicios. Por tanto, las dimensiones seleccionadas representan ampliamente el dominio de la gestión de la 
calidad en las empresas del sector servicios, tales dimensiones son: Planificación estratégica, gestión de procesos, Empowerment, benchmaking, mejora continua y orientación al cliente.

\section{Concepto de gestión del conocimiento}

En la literatura, hay muchas definiciones sobre gestión de conocimiento. Sin el deseo de hacer una búsqueda exhaustiva de las definiciones, se presentan algunas de ellas (Mejía, 2012).

a) La gestión del conocimiento es el proceso de manejar, continuamente, conocimiento de todas clases para encontrar necesidades existentes y emergentes, para identificar y explotar activos de conocimiento existentes y adquiridos y para desarrollar nuevas oportunidades (Quintas, Lefrere y Jones, 1997).

b) La gestión de conocimiento hace énfasis en facilitar y gestionar actividades relacionadas con el conocimiento tales como creación, captura, transformación y uso. Su función es planificar, implementar, operar y monitorear todas las actividades relacionadas con el conocimiento y con los programas requeridos para la gestión efectiva del capital intelectual (Wiig, 1997)

c) La gestión del conocimiento es "el proceso que continuamente asegura el desarrollo y aplicación de todo tipo de conocimientos pertinentes en una empresa, con objeto de mejorar su capacidad de resolución de problemas y así contribuir a la sostenibilidad de sus ventajas competitivas" (Andreu y Sieber, 1999).

d) La gestión del conocimiento es el proceso dinámico de convertir una práctica no-reflexiva en una reflexiva, aclarando las reglas que guían las prácticas, ayudando a dar una forma particular al entendimiento colectivo, y facilitando la emergencia de conocimiento heurístico (Tsoukas y Vladimirou, 2001).

En este trabajo se sigue el enfoque de Raghu y Vinze (2006 citado en Albacete, 2010), según los cuales la gestión del conocimiento puede ser definida como un conjunto cíclico de fases. Mientras cada una de estas fases puede ser una faceta única e independiente de la gestión del conocimiento, es la naturaleza interactiva de estas orientaciones la que explica la evolución continua del conocimiento y su gestión en las organizaciones. Estas fases son definidas como: creación de conocimiento, transferencia de conocimiento, acumulación y recuperación de conocimiento, y aplicación del mismo.

\section{Liderazgo}

En el ámbito de la gestión de la calidad, un liderazgo eficaz se demuestra a través de la correcta planificación e implantación de la innovación en la mejora de los procesos de la organización; la determinación de los procesos clave; la información a las personas de los programas estratégicos a desplegar; la supervisión, organización, gestión las disconformidades (Gorji y Emami, 2012) y; promoviendo el cambio cuando sea necesario, inspirando a los demás a seguirlos. En línea con esta última afirmación, Kotter (2001), hace referencia a la función de los líderes en la gestión del cambio, incluyendo la planificación, comunicación y gestión adecuada del mismo, siendo la dirección la responsable de revisar su efectividad.

Para Rao, Solis y Raghunathan (1999), la gestión de la calidad no sólo es responsabilidad de la alta dirección, sino que es una responsabilidad que no puede ser delegada. Así pues, la alta dirección debe aceptar su responsabilidad para liderar la calidad y proporcionar un soporte activo y puntual para construir una conciencia sobre la misma y lograr altos niveles de desempeño, para ello es imprescindible que los líderes sean capaces de percibir, conocer y comprender los principios básicos de la gestión de la calidad.

\section{Desempeño organizacional}

Una de las importancias en la que radica este constructo, es la medición sistemática de la misma, pues permite a la empresa conocer si las acciones que se están realizando logran los resultados esperados y si estos mejoran y se sostienen en el tiempo.

En el contexto de la organización más competitiva, se puede inferir que la importancia comienza con alinear las actividades empresariales a la visión estratégica para mejorar la comunicación interna y externa (Del Valle, 2016). Por otro lado, el desempeño organizacional es importante, ya que mejora el nivel de calidad 
de la organización, además encaminar un incentivo para adoptar o promover la innovación (Yamakawa y Ostos, 2011).

Según el análisis presentado por Richard, Devinney, Yip y Johnson (2009), el desempeño organizacional es el criterio más importante en la evaluación de las organizaciones, debido al uso generalizado del desempeño como una variable dependiente; en donde se presenta la posibilidad de conocer resultados, no solo cuantitativos, sino también cualitativos, siendo esta información de suma importancia para la organización (Yamakawa, et al, 2011).

Del Valle (2016), presenta que la importancia del desempeño organizacional en aspectos como los siguientes: (a) permitir a la organización conocer si las acciones que se están realizando logran los resultados esperados, (b) facilitar el benchmarking interno y externo para un futuro más competitivo, (c) alinear las actividades empresariales a la visión estratégica, (d) conducir al cambio organizacional, (e) mejorar el nivel de calidad de las organizaciones respecto al mercado y (f) promover la innovación.

\section{Hipótesis de investigación}

El propósito de este trabajo de investigación es presentar un modelo que contribuya a la mejora del desempeño organizacional de una universidad privada, en función a la opinión que tienen los directivos y docentes sobre las prácticas de gestión de calidad, gestión del conocimiento y liderazgo transformacional, con los resultados obtenidos durante el proceso de estudio.

Terminado la descripción general de los fundamentos que llevaron a definir la problemática de investigación, a través de la revisión de la literatura, y que sirvieron de soporte para el planteamiento de las hipótesis de investigación, esquematizándose así el modelo conceptual propuesto, como se muestra en la siguiente figura:

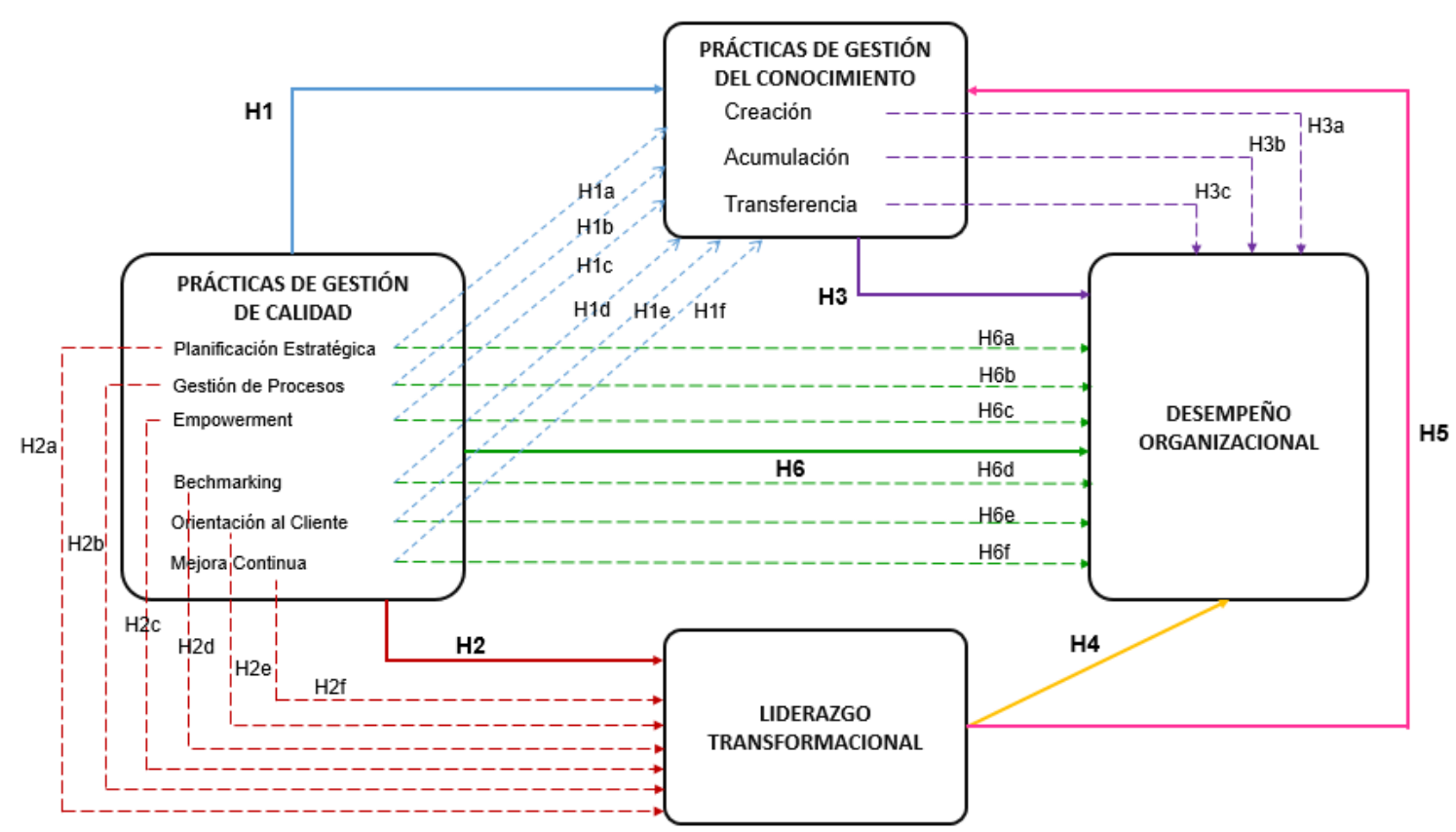

Figura 2. Diagrama del modelo teórico de las hipótesis de investigación

\section{Materiales y Métodos}

El diseño de investigación es correlacional múltiple con un enfoque transversal porque las variables fueron medidas en solo momento (Alarcón, 2008), ya que se hace uso de la solución de correlaciones múltiples simultáneas. Se contrasta empíricamente un modelo en función a las variables gestión de calidad, gestión del conocimiento y liderazgo transformacional con interdependencias y predictores del desempeño 
organizacional (Figura 3). A fin de cumplir con este propósito, el diseño se basa en el uso de modelos de ecuaciones estructurales, modelizado utilizando el programa informático de SPSS - AMOS versión 24.

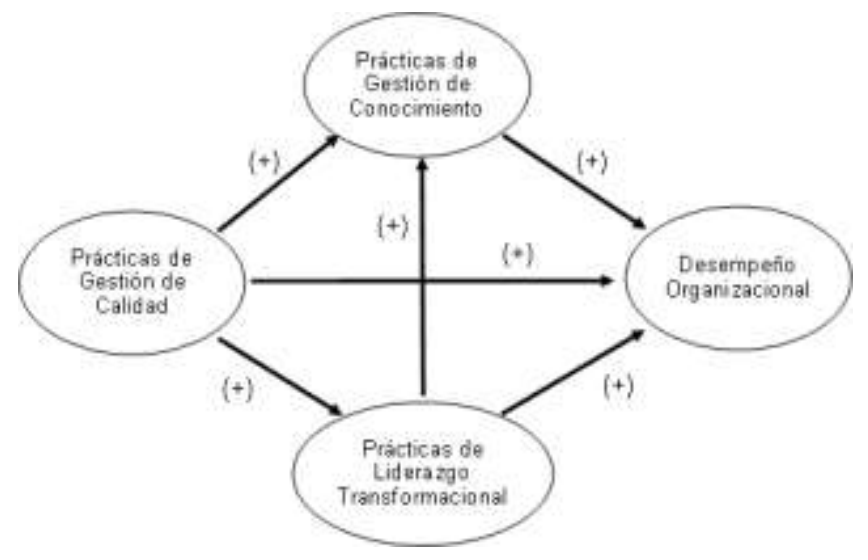

Figura 3. Modelo SEM del diseño de investigación

\subsection{Participantes}

Los participantes en el estudio fueron 222 profesionales, elegidos por el método de muestreo de tipo no probabilístico, que corresponde a lo que Elorza (2000) denomina muestreo determinístico, correspondiente al tipo de muestreo por conveniencia, ya que se procedió a establecer contacto con cada programa de estudio a través de los directores de escuelas de posgrado quienes indicaron a que docentes aplicar el instrumento, tomando en cuenta como criterio de inclusión a docentes en ejercicio laboral, docentes a tiempo completo y dedicación exclusiva; con la exclusión de docentes con contrato a tiempo parcial y licencia.

Los participantes en la presente investigación fueron personal directivo y docente de los tres campus de la universidad privada, siendo 144 hombres y 78 mujeres. El promedio de años en ejercicio laboral fue de 5.97 años. La cantidad de personal directivo es de 40 y personal docente 182, de todos estos 113 laboran en la sede central, 64 en el campus 02 y 45 en el campus 03 .

\subsection{Instrumentos}

Se plantea el análisis de un conjunto de hipótesis fruto de revisión teórica, basándose en los contrastes de relaciones causales entre las variables propuestas. Como se trata de una investigación con enfoque cuantitativo, se elaboró el cuestionario como el mecanismo más idóneo para obtener información de esta naturaleza.

Gestión de Calidad: El instrumento de prácticas de gestión de calidad fue adaptado y validado (según el análisis factorial) la propuesta de Albacete (2010) y Pérez (2009), de 42 ítems del instrumento inicial a 32 ítems (variables observadas), divididos en 6 dimensiones: Planificación estratégica, gestión de procesos, empowerment, benchmarking, orientación al cliente y mejora continua.

Gestión del Conocimiento: Para medir esta variable se utilizó la propuesta de Albacete Sáez (2010), adaptado al contexto de la universidad privada, en el cual se repite la misma operación del instrumento anterior. El instrumento consta de 14 variables observadas (ítems) dividido en tres dimensiones: Creación del conocimiento, acumulación del conocimiento y transferencia del conocimiento.

Liderazgo transformacional: Se utilizó el instrumento de Castro (2014), en el cual se repite la misma operación del instrumento anterior. El instrumento consta de 05 variables observadas (ítems).

Desempeño organizacional: Se utilizó la propuesta de Albacete Sáez (2010) y García (2011), adaptado al contexto de la universidad privada, en el cual se repite la misma operación del instrumento anterior. El instrumento consta de 07 variables observadas (ítems).

Una vez presentado la solicitud de inicio de la presente investigación con la anuencia de la alta dirección de la Universidad privada y la carta de autorización del comité de ética de la dirección de investigación de la Universidad privada, se procedió a fijar y explicar introductoriamente el desarrollo del plan de investigación 
con su instrumento, estableciendo su validez y confiabilidad y sobre todo llegar a las conclusiones y el aporte estratégico para el mejoramiento del desempeño de la organización.

\subsection{Análisis de datos}

Para el análisis descriptivo (medidas de tendencia central, variabilidad, entre otros) de las variables de estudio se utilizó el programa estadístico SPSS versión 24; ya también se usó el programa estadístico AMOS de SPSS versión 24 para el análisis factorial confirmatorio de los constructos de estudio y validación del modelo teórico con ecuaciones estructurales.

Cabe señalar que debido al tipo de variables utilizadas y a los tipos de relaciones planteadas en las hipótesis de investigación, se utilizó el modelo multivariante de ecuaciones estructurales, porque permite analizar las relaciones entre los conceptos teóricos, según Batista y Coenders (2000, citado por Burgos, 2010). Tal modelo permite trabajar simultáneamente con variables latentes y observadas, en el cual se puede incorporar la existencia de error de medida y, además, permite plantear distintas ecuaciones interrelacionadas.

\section{Resultados y discusión}

En primer lugar, se realizó el análisis estadístico descriptivo de los ítems de todas las variables de estudio. En según lugar se realizó la validación de la unidimensionalidad de las variables de estudio a través del análisis de estructura y fiabilidad de las escalas de medición. En tercer lugar, se aplicó el modelo multivariante de ecuaciones estructurales para comprobar la veracidad de las relaciones propuestas en las hipótesis de investigación, utilizando los índices de bondad de ajuste siguientes: (a) Ratio de verosimilitud de la chi cuadrada (X2) lo más pequeña posible y su nivel de significación p mayor o igual que .05 , (b) chi cuadrada normada (X2/gl) menor que 3, (c) Índice de bondad de ajuste (GFI) igual o mayor que .85, (d) Índice de bondad de ajuste comparativo (CFI) igual o mayor que .85 y (e) Raíz del residuo cuadrático promedio (RMSEA) igual o menor que .08. Las hipótesis fueron rechazadas para valores de significación p-valor menor o igual que .05. En cuarto lugar, se discuten las contribuciones de la investigación realizada luego del análisis desarrollado en relación a los constructos de este estudio.

\subsection{Ecuaciones del modelo estructural}

La especificación de un modelo estructural consiste básicamente en representar las hipótesis del modelo teórico en un sistema de ecuaciones estructurales, en el que se relacionan variables latentes endógenas y exógenas, siendo las variables latentes exógenas aquellas que no vienen explicadas por el modelo, mientras que las endógenas son las que el modelo pretende explicar. Con el fin de evitar errores de especificación de los modelos estructurales, es conveniente asegurarse de que no existen variables latentes endógenas y/o exógenas importantes omitidas en el modelo teórico (Albacete, 2010).

Para definir las ecuaciones correspondientes a los modelos de las variables de estudio e hipótesis de investigación, es necesario mostrar la figura 4 del modelo del objeto de contraste. 


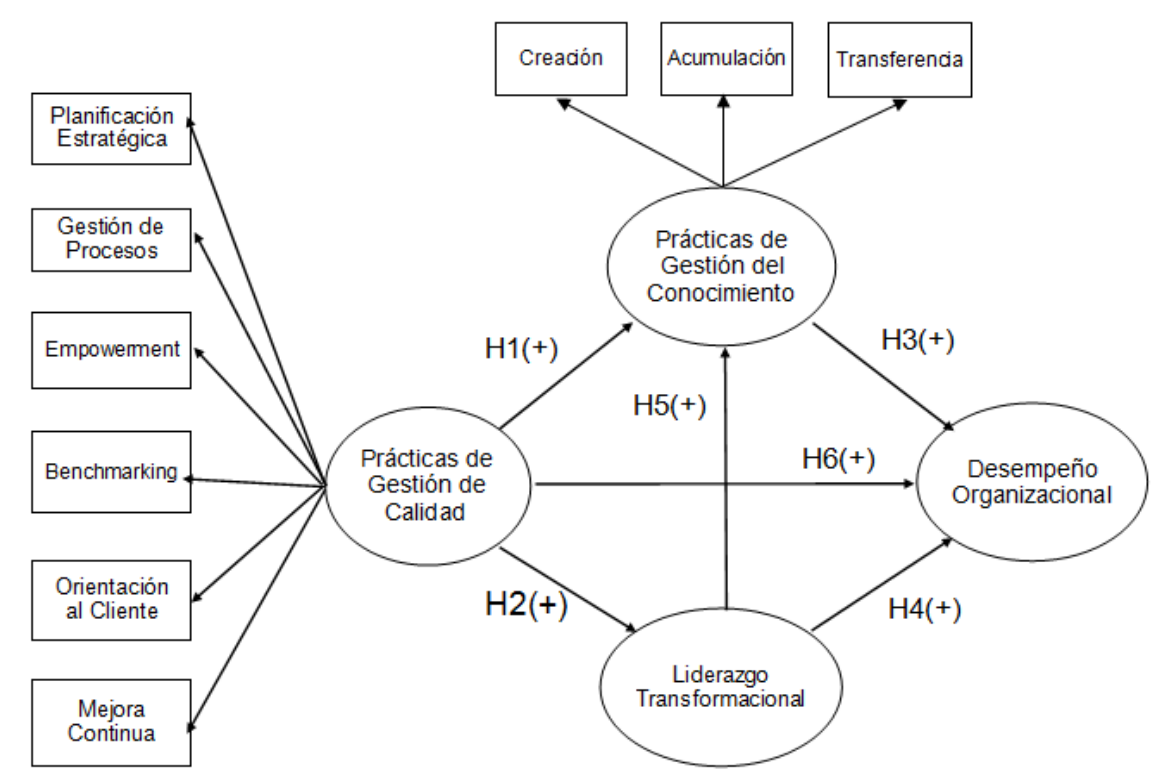

Figura 4. Diagrama del modelo teórico de contraste

A continuación, se muestran las ecuaciones correspondientes a las variables de estudio: Prácticas de gestión de calidad (tabla 1), gestión del conocimiento (tabla 2) y liderazgo transformacional (tabla 3).

Tabla 1

Ecuaciones del modelo de medida de las prácticas de gestión de calidad (C14)

\begin{tabular}{ll}
\hline Dimensión & Ecuación estructural \\
\hline Planificación estratégica (C1) & $\mathrm{C} 1=1 \mathrm{C} 14+\mathrm{E} 1$ \\
\hline Gestión de procesos (C2) & $\mathrm{C} 2={ }^{*} \mathrm{C} 14+\mathrm{E} 2$ \\
\hline Empowerment (C3) & $\mathrm{C} 3={ }^{*} \mathrm{C} 14+\mathrm{E} 3$ \\
\hline Bechmarking (C4) & $\mathrm{C} 4={ }^{*} \mathrm{C} 14+\mathrm{E} 4$ \\
\hline Orientación al cliente (C5) & $\mathrm{C} 5={ }^{*} \mathrm{C} 14+\mathrm{E} 5$ \\
\hline Mejora continua (C6) & $\mathrm{C} 6={ }^{*} \mathrm{C} 14+\mathrm{E} 6$ \\
\hline
\end{tabular}

Fuente: Elaboración propia

Tabla 2

Ecuaciones del modelo de medida de las prácticas de gestión del conocimiento (C15)

\begin{tabular}{ll}
\hline Dimensión & Ecuación estructural \\
\hline Creación del conocimiento $(\mathrm{C} 7)$ & $\mathrm{C} 7=1 \mathrm{C} 15+\mathrm{E} 7$ \\
\hline Acumulación del conocimiento $(\mathrm{C} 8)$ & $\mathrm{C} 8={ }^{*} \mathrm{C} 15+\mathrm{E} 8$ \\
\hline Transferencia del conocimiento $(\mathrm{C} 9)$ & $\mathrm{C} 9={ }^{*} \mathrm{C} 15+\mathrm{E} 9$ \\
\hline
\end{tabular}

Fuente: Elaboración propia

Tabla 3

Ecuaciones del modelo de medida del liderazgo transformacional (C16)

\begin{tabular}{ll}
\hline Dimensión & Ecuación estructural \\
\hline Visión compartida $(\mathrm{C} 10)$ & $\mathrm{C} 10=1 \mathrm{C} 16+\mathrm{E} 10$ \\
\hline
\end{tabular}




\begin{tabular}{ll}
\hline Motivación inspiradora (C11) & $\mathrm{C} 11=* \mathrm{C} 16+\mathrm{E} 11$ \\
\hline Toma de decisiones participativas (C12) & $\mathrm{C} 12=* \mathrm{C} 16+\mathrm{E} 12$ \\
\hline
\end{tabular}

Fuente: Elaboración propia

Enunciadas las ecuaciones de los modelos de medida de las variables de estudio, a continuación, en la tabla 4, se muestran las ecuaciones estructurales correspondientes a las hipótesis de investigación a contrastar.

Tabla 4

Ecuaciones estructurales de las hipótesis propuestas

\begin{tabular}{lc}
\hline Hipótesis & Ecuación estructural \\
\hline $\begin{array}{l}\text { H1: Las prácticas de gestión de la calidad (C14) tiene efecto } \\
\text { positivo en el desarrollo de gestión el conocimiento (C15) }\end{array}$ & \\
$\begin{array}{ll}\text { H5: El liderazgo transformacional (C16) tiene efecto positivo en } \\
\text { las prácticas de gestión del conocimiento (C15) }\end{array}$ & \\
$\begin{array}{ll}\text { H2: Las prácticas de gestión de la calidad (C14) tiene efecto } \\
\text { positivo en el liderazgo transformacional (C16) }\end{array}$ & $\mathrm{C} 16={ }^{*} \mathrm{C} 16+\mathrm{E} 15+\mathrm{E} 16$ \\
\hline
\end{tabular}

H3: Las prácticas de gestión del conocimiento (C15) tienen efecto

positivo en el desempeño organizacional (C13)

H4: El liderazgo transformacional (C16) tiene efecto positivo en el desempeño organizacional (C13)

H6:Las prácticas de gestión de la calidad (C14) tienen efecto positivo en el desempeño organizacional (C13)
Leyenda:
C: Constructo
E: Error
C13: Desempeño organizacional
C14: Gestión de la calidad
C15: Gestión del conocimiento
C16: Liderazgo transformacional

\subsection{Identificación y evaluación del modelo estructural}

Según Del Barrio y Luque (2000), se ha de trabajar con modelos que estén sobre identificados, es decir, modelos donde hay más valores conocidos que parámetros a estimar. En la tabla 46, se muestra la identificación del modelo complejo de estudio, donde el modelo cumple con la condición suficiente para la estimación misma del modelo en estudio.

\section{Tabla 51}

Resultados de cálculo de grados de libertad (modelo predeterminado)

\begin{tabular}{l|c}
\hline \multicolumn{1}{c|}{ Parámetro } & Valor \\
\hline $\begin{array}{l}\text { Number of distinct sample moments } \\
\text { (Número de momentos de muestra distintos): }\end{array}$ & 1769 \\
\hline Number of distinct parameters to be estimated & 208 \\
\hline
\end{tabular}


(Número de parámetros distintos a estimar):

\begin{tabular}{l|c}
\hline Degrees of freedom (grados de libertad=1769 - 208): & 1561 \\
\hline Chi-square (Ji cuadrado): & 2480.797 \\
\hline Probability level (probabilidad de error): & 0.000 \\
\hline
\end{tabular}

Considerando que la evaluación de la bondad de ajuste del modelo consiste en evaluar el grado en que los datos se han ajustado al modelo propuesto, en la figura 35, se presenta las estimaciones estandarizadas de los pesos factoriales que debe ser diferente de cero (coeficientes mayores de 0.5 y con nivel de significancia menor al 0.05); así como los índices de ajuste definidos para la evaluación del modelo. Sobre la base de análisis de los coeficientes estandarizados se determinó la validez factorial confirmatorio del modelo.

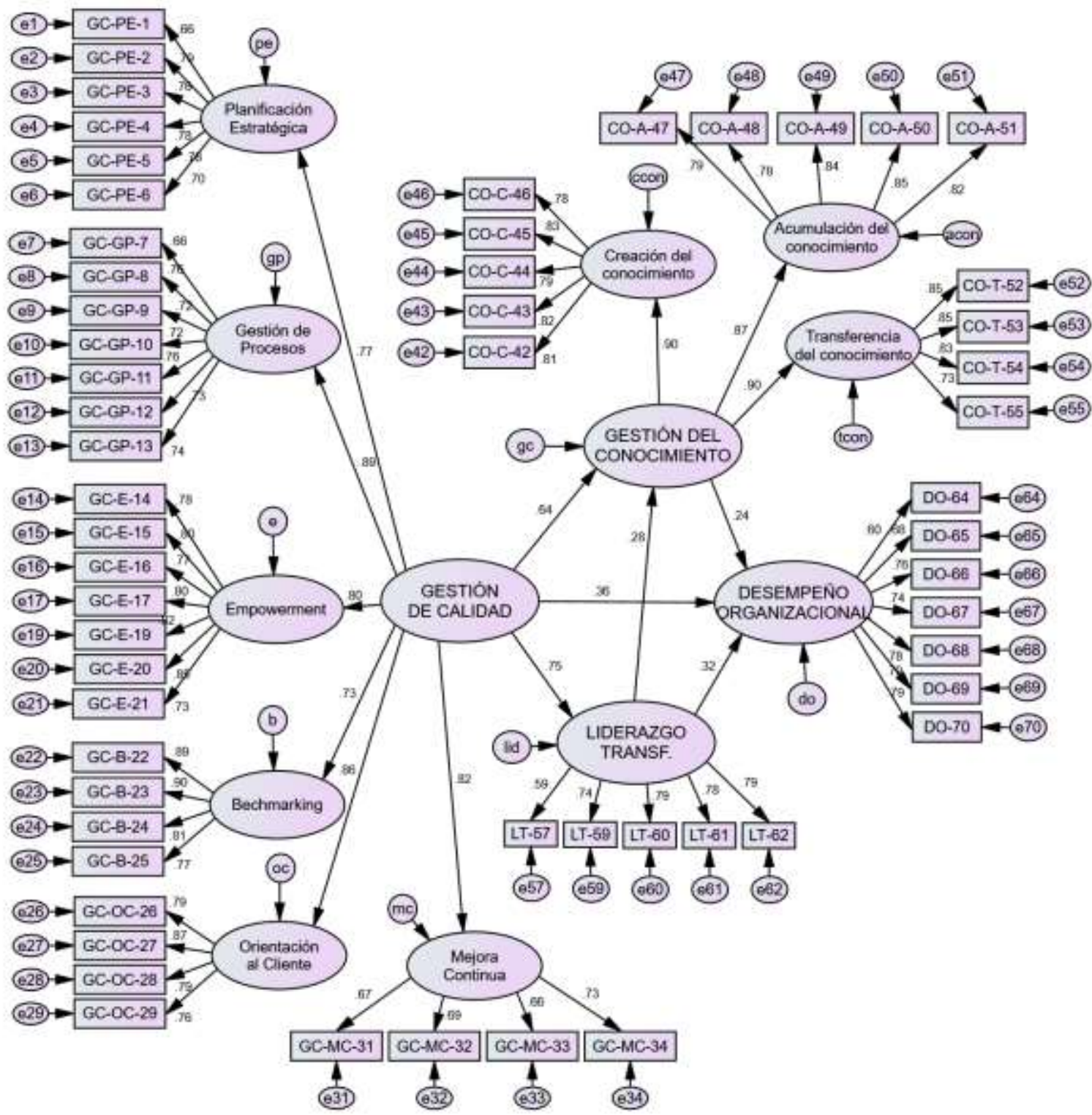

Figura 5. Análisis confirmatorio del modelo de la hipótesis general

La tabla 6, muestra las estadísticas de los índices de ajuste que permite calificar al modelo como como inaceptable, tomando como referencia el valor del RMSEA $=0.059>0.05$.

Tabla 6

Índices de ajuste del modelo estructural de la hipótesis general sin modificación 


\begin{tabular}{cccccc}
\hline$X^{2}$ & $X^{2} / d f$ & $C F I$ & $P C F I$ & GFI & RMSEA \\
\hline 2800.56 & 1.773 & 0.869 & 0.831 & 0.704 & 0.059 \\
\hline
\end{tabular}

Fuente. Análisis de data AMOS

Interesa entonces la modificación de índices para averiguar si es posible mejorar el ajuste del modelo. Cabe señalar que un modelo puede ser modificado artificialmente a la perfección mediante el análisis de los índices de modificación y consecuentemente mediante el establecimiento de las trayectorias sugeridas por los indicadores.

En el presente estudio, se ha probado la modificación de las rutas sugeridas, concluyendo que el modelo con modificación de índices mejoró sus índices de ajuste explicitas en la figura 6. Por tanto, el modelo presenta índices de ajuste de calidad que revelan una muy buena adaptación de la estructura modificada (tabla 7) y la identificación del modelo estructural ajustado (tabla 8).

Tabla 7

Índices de ajuste del modelo estructural de la hipótesis general sin modificación

\begin{tabular}{cccccc}
\hline$X^{2}$ & $X^{2} / d f$ & $C F I$ & $P C F I$ & GFI & RMSEA \\
\hline 2463.580 & 1.582 & 0.903 & 0.850 & 0.736 & 0.051 \\
\hline
\end{tabular}

Fuente. Análisis de data AMOS

Tabla 8

Resultados de cálculo de grados de libertad (modelo ajustado)

\begin{tabular}{l|c}
\hline \multicolumn{1}{c|}{ Parámetro } & Valor \\
\hline $\begin{array}{l}\text { Number of distinct sample moments } \\
\text { (Número de momentos de muestra distintos): }\end{array}$ & 1711 \\
\hline $\begin{array}{l}\text { Number of distinct parameters to be estimated } \\
\text { (Número de parámetros distintos a estimar): }\end{array}$ & 154 \\
\hline Degrees of freedom (grados de libertad=1711 - 154): & 1557 \\
\hline Chi-square (Ji cuadrado): & 2463.580 \\
\hline Probability level (probabilidad de error): & 0.000 \\
\hline
\end{tabular}

Fuente. Análisis de data AMOS

En la figura 6, se muestra la estructura del modelo ajustado permite estudiar y verificar la hipótesis general. 


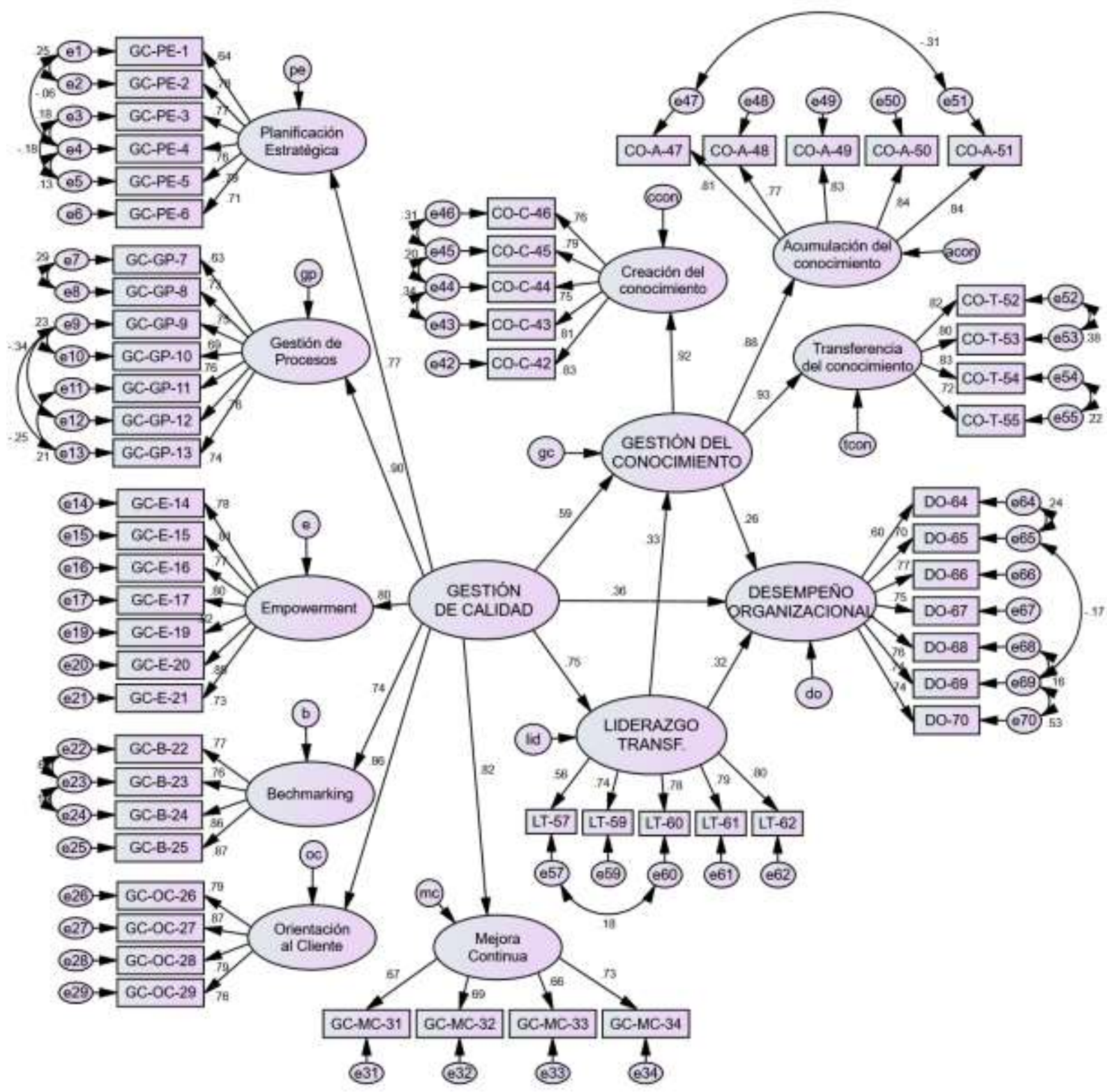

Figura 6. Análisis confirmatorio del modelo ajustado de la hipótesis general

La obtención de un nuevo modelo obliga a evaluar si la calidad del ajuste del modelo modificado es significativamente mejor que el modelo original. Las hipótesis a ser probadas son:

$$
\begin{aligned}
& H_{0}: x_{0}^{2}=x_{S}^{2} \\
& H_{1}: x_{0}^{2} \neq x_{S}^{2}
\end{aligned}
$$

Usando el valor de la estadística de chi-cuadrado y respectivos grados de libertad del modelo original y el modelo simplificado, la estadística de prueba es:

$$
x_{\text {dif }}^{2}=x_{0}^{2}-x_{S}^{2}=2480.797-2463.580=17.217
$$

Con $161-157=4$ grados de libertad, de acuerdo a la tabla de chi-cuadrado, para $\alpha=0.05$, se observa que: $x_{0.95 ;(4)}^{2}=9.488$

Siendo que: $x_{\text {dif }}^{2}=17.217>x_{0.95 ;(4)}^{2}=9.488$, se rechaza la hipótesis nula $\left(H_{0}\right)$, Por tanto, se puede concluir que el modelo ajustado se adapta mejor a la estructura de correlación observada entre los elementos de la muestra en estudio de la plantilla original. 


\subsection{Resultado de la hipótesis de investigación}

La tabla 9, tomando como referencia la muestra de estudio, revela que todas las relaciones establecidas en el modelo ajustado son estadísticamente significativas $(\mathrm{p}<0.05)$, concluyendo que de manera general para los fines de este trabajo el modelo presenta valores de ajuste razonables que permitirían validar la hipótesis general de investigación.

Tabla 2

Regression Weights: Standardized estimates e Unstandardized estimates - Hipótesis general

\begin{tabular}{lclrlllr}
\hline \multicolumn{2}{c}{ Correlación } & Estimate & U.E & S.E. & C.R. & p-valor \\
\hline G. Conocimiento & $<---$ & G. Calidad & 0.587 & 0.976 & 0.172 & 5.682 & $* * *$ \\
\hline Liderazgo Trasf. & $<---$ & G. Calidad & 0.754 & 1.309 & 0.169 & 7.760 & $* * *$ \\
\hline Desempeño Org. & $<---$ & G. Conocimiento & 0.262 & 0.249 & 0.111 & 2.245 & 0.025 \\
\hline Desempeño Org. & $<---$ & Liderazgo Trasf. & 0.318 & 0.290 & 0.086 & 3.384 & $* * *$ \\
\hline G. Conocimiento & $<---$ & Liderazgo Trasf. & 0.331 & 0.317 & 0.083 & 3.822 & $* * *$ \\
\hline Desempeño Org. & $<---$ & G. Calidad & 0.360 & 0.570 & 0.181 & 3.146 & 0.002 \\
\hline
\end{tabular}

S.E. - Standardized estimates; U.E. - Unstandardized estimates

*** is significantly different from zero at the 0,001 level (two-tailed)

Concluido el análisis estadístico de las variables de estudio que se centraron en el objetivo general de la investigación, importa ahora sintetizar los resultados obtenidos. En la tabla 51, figuran las principales conclusiones referentes con las hipótesis de investigación.

Tabla 10

Resumen de los resultados del contraste de hipótesis general

\begin{tabular}{ll}
\hline \multicolumn{1}{c}{ Hipótesis } & Resultado \\
\hline $\begin{array}{l}\text { H1. Las prácticas de gestión de la calidad tienen efecto positivo sobre las } \\
\text { prácticas de gestión del conocimiento en la universidad privada. }\end{array}$ & Significativa \\
\hline $\begin{array}{l}\text { H2: Las prácticas de gestión de la calidad tienen efecto positivo sobre las } \\
\text { prácticas del liderazgo transformacional en la universidad privada. }\end{array}$ & Significativa \\
\hline $\begin{array}{l}\text { H3: Las prácticas de gestión del conocimiento tienen efecto positivo sobre el } \\
\text { desempeño organizacional en la universidad privada. }\end{array}$ & Significativa \\
\hline $\begin{array}{l}\text { H4: Las prácticas del liderazgo transformacional tienen efecto positivo sobre el } \\
\text { desempeño organizacional en la universidad privada. }\end{array}$ & Significativa \\
\hline $\begin{array}{l}\text { H5: Las prácticas del liderazgo transformacional tienen efecto positivo sobre las } \\
\text { prácticas de gestión del conocimiento en la universidad privada. }\end{array}$ & Significativa \\
\hline $\begin{array}{l}\text { H6. Las prácticas de gestión de la calidad tienen efecto positivo sobre el } \\
\text { desempeño organizacional en la universidad privada. }\end{array}$ & Significativa \\
\hline
\end{tabular}

Fuente: Elaboración propia.

Los resultados del modelo analizado logran un ajuste satisfactorio a los datos de muestra general (fig. 6 y tabla 7). La razón $\mathrm{x}^{2} / \mathrm{gl}$ con un valor de 1.582 prueba la significación estadística del modelo. En cuanto a los índices de ajuste incremental, tanto el comparative fit index CFI=0.903 muestra un valor superior a 0,9. Para los índices de ajuste absoluto, el indicador goodness-of-fit index (GFI) toma un valor de 0,736 y el índice Root Mean Square Error of Approximation (RMSEA) muestra un valor de 0,051.

Luego de demostrar el buen ajuste del modelo, se analizaron las relaciones resultantes para verificar la validez de las hipótesis y así dictaminar su capacidad predictiva. En este sentido, se puede observar la fuerte 
y directa relación resultante entre la gestión de la calidad y la gestión del conocimiento (coeficiente estandarizado de 0,59), lo que permite dictaminar la validez de la hipótesis 1 . En cuanto a la hipótesis 2, se observa una relación fuerte y directa entre la calidad de la gestión de la calidad y el liderazgo transformacional (coeficiente estandarizado de 0,75 ), confirmando así su validez.

Por otro lado, se puede observar una fuerte y directa relación de la gestión del conocimiento y liderazgo transformacional, ambos, con el desempeño organizacional (coeficiente estandarizado de 0.26 y 0.32 ) confirmando las hipótesis 3 y 4 respectivamente.

La relación positiva y directa de la gestión de la calidad con el desempeño organizacional de verifica con el valor del coeficiente estandarizado de 0.36 , así también, se verifica la relación positiva e indirecta entre la gestión de la calidad y el desempeño organizacional, utilizando como mediadores en la relación la gestión del conocimiento y el liderazgo transformacional. Al respecto, se obtiene un efecto indirecto y positivo de $0.59 * 0.26=0.15$ para la relación gestión de la calidad $\rightarrow$ gestión del conocimiento $\rightarrow$ desempeño organizacional y de $0.75 * 0.32=0.24$ para la relación gestión de la calidad $\rightarrow$ liderazgo transformacional $\rightarrow$ desempeño organizacional. El efecto total entre la gestión de la calidad y el desempeño organizacional por el uso de variables moderadoras es de $0.36+0.15+0.24=0.75$, significativamente superior a la relación que existe entre la gestión de la calidad y el desempeño organizacional. En consecuencia, se confirman las hipótesis descritas, así como la significación estadística de las relaciones indirectas.

Finalizado el análisis de los resultados del análisis estadístico de hipótesis de investigación definidas en el modelo conceptual propuesto (modelo de relaciones), se establece en el capítulo siguiente las principales conclusiones del análisis.

\subsection{Discusión}

Después de haber analizado cómo las prácticas de gestión de la calidad repercuten en las prácticas de gestión del conocimiento y el liderazgo transformacional, se puede afirmar también que la misma no sólo tiene efecto de manera directa en el desempeño organizacional, sino que, además, puede contribuir a la mejora de las prácticas de gestión del conocimiento y liderazgo transformacional. Se ha contrastado también cómo la intervención de estas variables ejerce un efecto mediador en la relación entre la gestión de la calidad y el desempeño organizacional.

Al contrastar la hipótesis de investigación se corrobora diversas investigaciones realizadas en las que las prácticas de gestión de la calidad, gestión del conocimiento y liderazgo proporcionan a las organizaciones instrumentos muy valiosos para crear y mantener ventajas competitivas que se traducen en mejores resultados acorde a los propósitos internos y demandas del entorno.

Todo esto nos permite señalar que la implantación de las prácticas de gestión de la calidad en una organización educativa como la universidad privada asegura un clima y cultura propicia para el desarrollo de la gestión del conocimiento, la cual, es especialmente determinante en un contexto donde la intangibilidad de los servicios provoca la existencia de un alto volumen de capital intangible que es necesario gestionar; así también, las prácticas de gestión de la calidad resulta fundamental en apoyar los esfuerzos gerenciales a través de la influencia en un liderazgo transformacional que contribuya a mejorar el desempeño organizacional.

La relación entre gestión de la calidad y gestión del conocimiento, indican que muchas de las actividades que son llevadas a cabo bajo un escenario de gestión de la calidad, pueden ser consideradas como medios para obtener información, la cual es acumulada y procesada por la organización y puede ser usada para crear nueva información y conocimiento (Nonaka, 1994). No obstante, según el análisis desarrollado, la dimensión empoderamiento de la variable gestión de la calidad resulto no ser significativa mostrando una probabilidad de error del $34.2 \%$, que es superior al $5 \%$, esto no significa asumir una posición contraria a la literatura, mas es una opinión de la unidad de estudio (directivos y docentes) en el contexto del que hacer universitario.

Igualmente, la gestión de la calidad está fuertemente relacionada con la práctica de un liderazgo transformacional en todos los niveles de la organización, siendo este un elemento dinamizador en un marco de sistema de gestión de la calidad.

Globalmente se puede afirmar que la implantación de las prácticas de gestión de la calidad en organizaciones de servicios, en concreto, educativas, permite crear un clima propicio para el desarrollo de 
prácticas de gestión del conocimiento y como ente dinamizador el liderazgo transformacional. Igualmente podemos afirmar que la gestión del conocimiento influye positivamente sobre el desempeño organizacional. Por otro lado, podemos indicar que la creación de conocimiento es la práctica más relevante dentro del grupo de prácticas de gestión del conocimiento, pues dicha práctica influye positivamente sobre el desempeño organizacional.

Con los resultados obtenidos podemos plantear aspectos que ayuden a la organización a alcanzar una situación esperada con resultados objetivos, entre las cuales señalamos: a) Las estrategias de gestión de la calidad, gestión del conocimiento y liderazgo, son estrategias que proporcionan a las organizaciones mejoras en su desempeño, resultando especialmente útiles en los entornos actuales caracterizadas por su alta dinamicidad; y b) La gestión de la calidad, se ha configurado como una estrategia básica en el sector educativo, donde el cliente es cada vez más exigente. No obstante, no basta simplemente con desarrollar prácticas de gestión de la calidad orientadas a la satisfacción del cliente y la mejora continua, sino que estas deberían considerar su potencial para ser soporte de los procesos de gestión del conocimiento y liderazgo, pues además de contribuir a los objetivos propios de la gestión de la calidad, estas prácticas suponen una importante infraestructura para el desarrollo posterior de prácticas como la gestión del conocimiento y el liderazgo.

\section{Conclusiones}

De modo general, el análisis multivariante denominado modelo de ecuaciones estructurales, utilizando el método de máxima verosimilitud, alcanzó los valores de criterios de ajuste seleccionados, con la evaluación de seis indicadores jerarquizados $(\mathrm{X} 2=2463.580, \mathrm{X} 2 / \mathrm{df}=1.582, \mathrm{CFI}=0.903, \mathrm{PCFI}=0.850, \mathrm{GFI}=0.736$ y RMSEA=0.051), siendo las seis relaciones de tipo causal totalmente significativas ( $\mathrm{p}$-valor $<0.05$ ), con lo cual se concluye que el modelo teórico propuesto posee validez empírica que orienta la mejora del desempeño organizacional por efecto de las prácticas de gestión de calidad, gestión del conocimiento y liderazgo transformacional en la universidad privada, año 2016.

Por otro lado, como consecuencia del análisis, podemos señalar también que la variable clave del modelo estructural final e influyente en el desempeño organizacional fue la gestión de la calidad, el cual tiene un efecto directo e indirecto con todas las variables de este modelo, mostrando un coeficiente estandarizado de 0.36 ( $\mathrm{p}$-valor=0.002) relativamente mayor a los coeficientes de las variables gestión del conocimiento y liderazgo transformacional cuyos coeficientes son 0.262 (p-valor=0.025) y 0.331 ( $\mathrm{p}$-valor=0.00) respectivamente.

\section{Recomendaciones}

Se recomienda que la universidad privada fortalezcan, establezcan o mejoren la administración de las prácticas de gestión de calidad, gestión del conocimiento y liderazgo en todos los niveles, con el fin de alcanzar los objetivos propuestos como organización y obtener mejores resultados.

Aplicar el instrumento a una muestra mayor que la que se aplicó actualmente.

Realizar nuevas investigaciones aplicando diversos métodos (ML, ULS y SLS) disponibles para resolver ecuaciones estructurales. Al aplicar diversos métodos, se podrá evaluar el efecto que se puede tener sobre los índices de ajuste de modelos sobre los coeficientes estandarizados, sobre los coeficientes no estandarizados y sobre la varianza explicada de los constructos endógenos.

Agregar nuevos constructos exógenos, para tratar de elevar la varianza explicada de los constructos exógenos desempeño organizacional. 


\section{Referencias}

Ahire, S. L.; Landeros, R. y Golhar, D. Y. (1995), “Total quality management: a literature review and an agenda for future research", Production and Operations Management, Vol. 4, No. 3, pp. 277-306.

Alarcón, R. (2008). Métodos y Diseños de Investigación del Comportamiento. Lima, Perú: Editorial Universitaria - Universidad Ricardo Palma.

Alarcón, S. (2013). Gestión Educativa y Calidad de la Educación en Instituciones privadas en Lima Metropolitana. (Tesis de maestría). Universidad de San Martin de Porres, Lima-Perú.

Alavi, M. y Leidner, D. E. (2001), “Knowledge management and knowledge management systems: conceptual foundations and research issues”, MIS Quarterly, Vol. 25, No. 1, pp. 107-136.

Albacete, Carlos A. (2010). Influencia de las prácticas de gestión de la calidad sobre la gestión del conocimiento y la innovación en los servicios: el caso de las empresas hoteleras (Tesis de doctorado). Universidad de Granada, Granada, España.

Alcade, P. (2010). Calidad. Madrid, España: Paraninfo.

Andreu, R. y Sieber, S. (1999). La Gestión Integral del Conocimiento y del Aprendizaje. Economía Industrial. $\mathrm{N}^{\circ}$ 326: 63-72.

Arelis, M. (2016). Modelo de evaluación institucional, basado en el enfoque sistémico, para valorar la calidad de la gestión en la Institución Educativa No 80292 Daniel Alcides Carrión De Pango - Otuzco. (Tesis de Maestría). Universidad Nacional de Trujillo, Trujillo -Perú.

Bernal, César A. (2006). Metodología de la Investigación. Segunda edición. Pearson Educación: Colombia. Burgos, D. (2010). Niveles de integración de la fe de los maestros de colegios secundarios adventistas de el salvador: algunos factores predictores. (Tesis doctoral). Universidad de Montemorelos Montemorelos, México.

Caballano, J. (2012). El prisma. Obtenido de http://www.elprisma.com/apuntes/administracion_de_empresas/gestiondelacalidad/

Camisón, C., Cruz, S., \& González, T. (2006). Gestión de la Calidad. Conceptos, Enfoques, Modelos y Sistemas. Madrid, España: Pearson Educación S.A.

Camisón, C.; Boronat, M.; Villar, A.; y Puig, A. (2009). Sistemas de gestión de la calidad y desempeño: importancia de las prácticas de gestión del conocimiento y de I+D. Revista Europea de Dirección y Economía de la Empresa, vol. 18, núm. 1 (2009), pp. 123-134.

Castro, L. (2014). Liderazgo Transformacional y Aprendizaje Organizacional del Personal Directivo en la Universidad Nacional del Callao, 2013 (Tesis doctoral). Universidad san Martin de Porres, Lima, Perú. Cevalllos, D. (2014). La Calidad Educativa en la realidad Universitaria Peruana frente al Contexto Charaja, F. (2009). El MAPIC en la metodología de investigación. Segunda edición. Sagitario Impresiones: Puno, Perú. 
David, F. (2013). Conceptos de Administración Estratégica. 14 Edición. México. Pearson Educación. 2013. pp. 5-13-15-56-59-81-159.

Del Barrio, S. y Luque, T. (2000). Análisis de ecuaciones estructurales. En T. Luque, (Ed.), Técnicas de análisis de datos en investigación de mercados (pp. 489 - 557). Madrid, España: Ediciones Pirámide.

Del Castillo, C. y Vargas, B. (2009). El proceso de gestión y el desempeño organizacional: una aproximación a la nueva gestión pública desde el ámbito de los gobiernos locales. Cuadernos de Difusión, 14(26), 57-80.

Del Valle, Jair (2016). Modelo Asociativo entre Factores Determinantes del Desempeño Organizacional y la Satisfacción de los Públicos en Instituciones Educativas (Tesis de doctorado). Universidad de Montemorelos, Nuevo León, México.

Díaz Jaimes, M. P. y Ortíz Pimiento, N. R. (2012). Revisión de modelos de madurez: estrategia de evaluación del desempeño para empresas de manufactura. UIS Ingenierías, 11(1), 55-72.

Douglas, T. J. y Fredendall, L. D. (2004), “Evaluating the Deming management model of total quality in Gálvez, E. y Pérez, D. (2011). Cultura organizacional y rendimiento de las MIPYMES de mediana y alta tecnología: un estudio empírico en Cali, Colombia. Cuad. admon.ser.organ. Bogotá (Colombia), 24 (42): $125-145$.

García, I. (2013). Percepción Gerencial de los Factores que Contribuyen con el Desempeño en las Organizaciones de Negocios. Revista de Investigación Universitaria. Vol. 2 (2): 37-50.

García, V.; Jiménez, M. \& Gutiérrez, L. (2012). Transformational leadership influence on organizational performance through organizational learning and innovation. Journal of Business Research. Doi:10.1016/j.jbusres.2011.03.005.

García, V.; Lloréns, F. \& Verdú, A. (2007). The Effects of Transformational Leadership on Organizational Performance through Knowledge and Innovation. British Journal of management, Vol 19 (4), 299-319. DOI: $10.1111 /$ j.1467-8551.2007.00547.x

Gorji, M.; Emami, A. (2012): “Self-assessment of organization's performance with regard to enablers criteria Excellence Model (EFQM)”, Research Journal of Applied Sciences, Engineering and Technology, vol. 4, núm. 17, pp. 3048-3055.

Hassan, M., Shaukat, S. \& Saqib, M. (2013). Relationship between TQM Elements and Organizational Performance: An Empirical Study of Manufacturing Sector of Pakistan. Pakistan Journal of Commerce and Social Sciences, Vol. 7 (1), 01-18.

Hernández Sampieri, R., Fernández Collado, C. y Baptista Lucio, M. (2014). Metodología de la investigación (6ta ed.). México: McGraw Hill.

Hussain, T., Younis, A. (2015). Quality Management Practices And Organizational Performance: Moderating Role Of Leadership. Sci.Int. Lahore, 27(1), 517-522. 
Jarour, S. (2013). Quality Management Practices and Their Impact on Organization Performance.

International Journal of Management Sciences and Business Research, Vol. 2 (6).

Juran, J. M. (1989). Juran on leadership for quality: an executive handbook. Nueva York: Free Press.

Jyoti, J.; Bhau, S. (2015). Impact of Transformational Leadership on Job Performance: Mediating Role of Leader-Member Exchange and Relational Identification. DOI: 10.1177/2158244015612518.

Kamarulzaman, R. y Mohamed, S. (2016). Transformational Leaders and Organizational Performance. Proceedings of the 1st AAGBS International Conference on Business Management 2014 (AiCoBM 2014), DOI 10.1007/978-981-287-426-9_57.

Kiprotich, W. (2014). Effect of Total Quality Management Practices on Organizational Performance in Kenya: A Case Of Tertiary Institutions in Uasin Gishu County. (Tesis de maestría). Kisii University, Kenia.

Knight, G. (2012). Las implicaciones de la filosofía de la educación adventista: la educación redentora (segunda parte). Revista de Educación Adventista, 33, 24-41.

Koontz, H. y O`Donnell, C. (1959). Principles of management. New York: McGraw-Hill.

Kotter, J. P. (1988): The leadership factor, Free Press, New York.

Laohavichien, T.L., Fredendall R.D. y Stephen C. (2011). Leadership and quality management practices in Thailand. International Journal of Operations \& Production Management, 31, 10, 1048-1070.

Lee D. (2015). The effect of operational innovation and QM practices on organizational performance in the healthcare sector. International Journal of Quality Innovation, 1(8).

Lee, C. C. y Yang, J. (2000), “Knowledge value chain”, Journal of Management Development, Vol. 19, No. 9, pp. 783-793.

McKeen, J., Zack, M., \& Singh, S. (2006). Knowledge Management and Organizational Performance: An Exploratory Survey. Proceedings of the 39th Hawaii Conferencia Internacional sobre las Ciencias del Sistema.

Medina, A.G. (2005). Gestión por procesos y creación del valor público. Un enfoque analítico. Santo Domingo: Nueva Gestión pública.

Mejía, M. (2008). Modelo de gestión del conocimiento para las empresas de la industria del software peruana (Tesis doctoral). Universidad Nacional Mayor de San Marcos. Lima, Perú.

Ministerio de Educación - MINEDU (2015) Política de aseguramiento de la calidad de la educación superior universitaria. Lima: El Peruano 26 setiembre.

Munizu M. (2013). The Impact of Total Quality Management Practices towards Competitive Advantage and Organizational Performance: Case of Fishery Industry in South Sulawesi Province of Indonesia. Pakistan Journal of Commerce and Social Sciences, Vol. 7 (1), 184-197.

Nonaka, I. (1994), “A dynamic theory of organizational knowledge creation”, Organization Science, Vol. 5, No. 1, pp. 14-37. 
Nonaka, I. y Takeuchi, H. (1999). La organización creadora de conocimiento: cómo las compañías japonesas crean la dinámica de la innovación. México: Oxford University Press.

Pérez Zapata, J. y Cortés Ramírez, J. A. (2009). Medición y validación del desempeño organizacional como resultado de acciones de aprendizaje. Revista Ciencias Estratégicas, 17(22), 251-271.

Pérez, María (2009). La Relación entre la Tecnología de la Información, Las Prácticas de Gestión de la Calidad y el Desarrollo de las Capacidades Dinámicas: Una Aproximación Empírica (Tesis de doctorado). Universidad de Granada, Granada, España.

Pola, A. (1999). Gestión de la Calidad. Barcelona: Marcombo S.A.

Powell, T. C. (1995), "Total quality management as competitive advantage: a review and empirical study", Strategic Management Journal, Vol. 16, No. 1, pp. 15-37.

Pulido, A. (2011). Fundamentos de Gestión Empresarial. Enfoque basado en Competencias. México D.F.: Mc Graw - Hill.

Quintas, P.; Lefrere, P. y Jones, G. (1997), “Knowledge management: a strategic agenda”, Long Range Planning, Vol. 30, No. 3, pp. 385-391.

Raghu, T. S. y Vinze, A. (2007), “A business process context for knowledge management”, Decision Support Systems, Vol. 43, No. 3, pp. 1062-1079.

Ramos López, M. A. (2005). Mujeres y liderazgo. Valencia: Universidad de Valencia.

Rao, S. S.; Solis, L. E. y Raghunathan, T. S. (1999), “A framework for international quality management research: development and validation of a measurement instrument”, Total Quality Management, Vol. 10, No. 7, pp. 1047-1075.

Rasula, J., Bosilj, V. \& Indihar, M. (2012). The Impact of Knowledge Management on Organisational Performance. Economic and Business Review, Vol. 14 (2), 147-168.

Richard, P. J., Devinney, T. M., Yip, G. S. y Johnson, G. (2009). Measuring organizational performance: Towards methodological best practice. Journal of Management, 35(3), 718-804. doi:10.1177/0149206308330560.

Ruiz, J. I. (2012). Teoría y Práctica de la Investigación Cualitativa. Bilbao: Universidad de Deusto. Saad, G.H.; Siha, S. (2000). "Managing quality: critical links and a contingency model”. International Journal of Production and Operations Management, Vol. 20, nº10, pág.1146-1163.

Salami, C., Obgeta, M. (2015). Knowledge Management and the Organizational Performance: A Study Of Mtn Nigeria Limited, Asaba - Delta State. (Tesis de doctorado). Delta State University, Abraka.

Saravanan, R. y Rao, K. S. P. (2006), "Development and validation of an instrument for measuring total quality service”. Total Quality Management \& Business Excellence, Vol. 17, No. 6, pp. 733-749. Toro, J. (2012). Gestión interna la calidad en instituciones de educación superior. Providencia, Chile; Ril, CINDA. 
Tsoukas, H. y Vladimirou, E. (2001). What is Organizational Knowledge? Journal of Management Studies. Vol 38, $\mathrm{N}^{\circ}$ 7: 973-993.

Turpo, J. (2014). Cultura organizacional, imagen institucional y calidad de los servicios educativos según la percepción de los alumnos, padres, docentes y público externo de la universidad peruana unión. (Tesis de doctorado). Universidad Peruana Unión. Lima- Perú.

White, E. (1978). El hogar cristiano (7a ed.). Mountain View, CA: Pacific Press.

White, E. (2001). Ministerio médico. México: Asociación Publicadora Interamericana.

White, E. (2003). Liderazgo cristiano. Silver Spring, MD: White Estate.

Wiig, K. M. (1997), “Integrating intellectual capital and knowledge management”, Long Range Planning, Vol. 30, No. 3, pp. 399-405.

Wiig, K. M. (1997). “Integrating intellectual capital and knowledge management”, Long Range Planning, Vol. 30, No. 3, pp. 399-405.

Yamakawa Tsuja, P. y Ostos Mariño, J. (2011). Relación entre innovación organizacional y desempeño organizacional. Universidad y Empresa, 21, 93-115. 